\title{
Peroxisome proliferator-activated receptors: Targets for the treatment of metabolic illnesses (Review)
}

\author{
RODRIGO MOORE-CARRASCO ${ }^{1}$, MAURICIO POBLETE BUSTAMANTE ${ }^{2}$, \\ OSCAR GONZÁLEZ GUERRA ${ }^{3}$, ELBA LEIVA MADARIAGA ${ }^{1}$, VERONICA MUJICA ESCUDERO ${ }^{1}$, \\ CLAUDIO ARANGUEZ ARELLANO ${ }^{1}$ and IVÁN PALOMO ${ }^{1}$ \\ ${ }^{1}$ Departamento de Bioquímica Clínica e Inmunohematología, ${ }^{2}$ Instituto de Biología Vegetal y Biotecnología, \\ Universidad de Talca, Talca; ${ }^{3}$ Hospital San Agustín, Collipulli, Chile
}

Received November 29, 2007; Accepted January 15, 2008

\begin{abstract}
Peroxisome proliferator-activated receptors (PPARs) belong to a family of transcription factors of which three isotypes, PPAR $\alpha, \operatorname{PPAR} \delta(ß)$ and PPAR $\gamma$, are known. These play a central role in regulating intermediate metabolism and in incidences of inflammation. In recent years, a greater understanding of their mechanisms of action and their effects, principally in the management of cardiovascular disease, has been achieved. PPAR agonists, catalysts and agents have been used since the 1990s, when it was confirmed that fibrates possess lipid modifying properties when selectively activating PPAR $\alpha$. In addition, thiazolidinediones, structures analogous to fibrates, showed PPAR $\gamma$ activity with an insulin-sensitizing effect, leading to their use in the control and even prevention of diabetes mellitus type 2. Currently, studies are oriented to the development of agents that activate multiple PPAR isoforms - not only dual (PPAR $\alpha / \gamma)$, but also PPAR pan-agonists $(\alpha / \gamma / \delta)$. The purpose of this review is to explain the mechanisms of the molecular action and the effects of PPAR agonists, and also to analyze existing and current studies concerning their use in cardiovascular and metabolic illnesses.
\end{abstract}

\section{Contents}

1. Introduction

2. Characteristics of PPARs

3. Metabolism and PPAR agonists

4. Fibrates and diabetes

5. Beyond glycemic control

Correspondence to: Dr Rodrigo Moore-Carrasco, Department of Clinical Biochemistry and Immunohematology, Faculty of Health Sciences, University of Talca, P.O. Box 747, Talca, Chile E-mail: rmoore@utalca.cl

Key words: peroxisome proliferator-activated receptor, cardiovascular diseases, diabetes mellitus, fibrates, thiazolidinediones
6. Potential applications of PPAR agonists

7. Conclusions

\section{Introduction}

In complex organisms, human beings included, life depends on the metabolic regulation of lipids and carbohydrates including the organization of, and adequate response to, the surrounding environment, such as the intake of food, stress, physical activity and temperature. In order to ensure continual adaptation to these environmental factors and the maintenance of the necessary energy levels to make them compatible with life, close coordination is also required in a series of metabolic reactions at different levels, such as in the hypothalamus, muscular cells, adipose tissue, pancreas and liver. This involves the participation of a large number of metabolic mediators, including transcription factors.

Transcription factors are proteins that unite with genetic zones, called promotion regions, capable of controlling the expression of genes. They can be activated or repressed by extracellular or intracellular stimuli; for example, by membrane receptors that set in motion a chain of reactions with the purpose of carrying specific information through the cytoplasm and finally to the transcription factor, thus regulating genetic expression.

A family of transcription factors known as peroxisome proliferator-activated receptors (PPARs) plays a central role in the regulation of the storage and catabolization of fatty acids. These receptors are found united to the nuclear membrane and are thus named because they are capable of activating the proliferation of peroxisomes in rats (1). Moreover, the identification of ligands for the PPARs has provided the ideal approach to the study of the homeostasis of lipids at the molecular level. In fact, once activated by the ligands, whether synthetic or made by the organism itself, PPARs control a large number of genes involved in many pathways related to intermediate metabolism. To date, three PPAR isotypes have been identified, PPAR $\alpha$, PPAR $\delta(ß)$ and finally PPAR $\gamma$, for which 2 isoforms, PPAR $\gamma 1$ and PPAR $\gamma 2$, have been found. The location of the PPAR $\alpha$ gene is on chromosome 22 of the region 22q12-q13.1; PPAR $\delta$ is located on chromosome 6 in 
position 6p21.1-p21.2, and PPAR $\gamma$ on chromosome 3 in position $3 \mathrm{p} 25$ (2).

\section{Characteristics of PPARs}

The different types of PPARs have common structures with functional domains. The classification of the genes reveal that they have a common structure possessing codifying exons: one exon for the $\mathrm{A} / \mathrm{B}$ domain of the $\mathrm{N}$-terminal, two exons for the DNA-binding domain, an exon for each one of the 'zinc fingers', an exon for the hinge region and two exons for the ligand-binding domain (LBD). The $\mathrm{A} / \mathrm{B}$ domain can undergo modification, especially phosphorylation, which can activate these transcription factors (3).

PPARs form heterodimers with another nuclear receptor, 9 cis-retinoic acid receptor, which binds with DNA sequences containing two hexanucleotides known as PPAR response elements (PPREs). The nucleotide sequence is AGGTCA, separated by a nucleotide; this sequence is called direct repeat, DR-1. Such sequences have been found in numerous PPARinduced genes, such as acyl-CoA synthetase, acyl-CoA oxidase and undesacoplant protein 1 (4).

PPARs can be activated by different types of compounds, called ligands, that are bound in the LBD. A characteristic of ligands is that they are all hydrophobic. There are natural ligands (fatty acids) and synthetic ones, among which are found drugs, such as thiazolidinediones (TZDs) and fibrates, currently being used in different therapeutic lines. These will be discussed later.

It has been found that PPAR distribution and activity differs according to tissue. PPAR $\alpha$ is expressed in tissues with a high mitochondrial and peroxisomal content, such as in the heart, liver, muscles and kidneys, where the fatty acids are catabolized. It is also expressed in monocytes/macrophages and foam cells. PPAR $\gamma$ is abundantly expressed in adiposites and macrophages, and can also be found in skeletal muscle and thyroids. It is related to the differentiation of adiposites, the storing of lipids and glucose homeostasis. Finally, PPAR $\delta$ is found expressed in the brain, adiposites, skin and skeletal muscle, among others (5).

\section{Metabolism and PPAR agonists}

PPAR $\alpha$ plays a crucial role in the intracellular metabolism of lipids, regulating the expression of proteins related to the transport and $\beta$-oxidation of fatty acids in the liver, predominantly. PPAR $\alpha$ agonists stimulate the fatty acid transport protein in the liver, which allows for the uptake of fatty acids into the interior of the organ (2). Moreover, they promote B-oxidation for inducing the key enzymes of this process, such as acyl-CoA dehydrogenase, enoyl CoA hydratase and acylCoA acetyltransferase (6). The finding that all these enzymes are induced in the presence of the PPAR $\alpha$ agonist shows the importance of these nuclear factors in the metabolism of fatty acids and energy homeostasis. In fact, knockout mice do not produce transcription activation of the enzymes and proteins associated with the transport of fatty acids after fibrate stimulation, which causes them to have intrahepatic fat accumulation following a normal diet $(7,8)$. They are also incapable of responding to fasting states, resulting in hypo- acetonemia, which is associated with severe hypothermia and hypoglycemia (7). PPAR $\alpha$ is associated with the inflammatory process as knockout mice show a prolonged inflammatory response (9-11). Furthermore, these mice present alterations in their cardiac function (12). On the other hand, they show protection against insulin resistance induced by high-fat diets (13).

PPARs are expressed in the great majority of the cells that make up the vascular system, endothelial cells, monocytes/ macrophages, foam cells and T linfocytes (14). The first clue that PPAR $\alpha$ is related to atherogenesis is the fact that fibrates are capable of reducing atherosclerotic plaque formation, regardless of changes in the levels of lipids in the blood (15). Synthetic PPAR $\alpha$ agonist and fenofibrates decrease the expression of VCAM-1 (16). This control is due in part to the suppression of $\mathrm{NF \kappa B}$, the key factor in the activation of the VCAM-1 promoter. The anti-inflammatory action of the PPARs occurs through the suppression of NFKB; PPARs keeps $\mathrm{NF} \kappa \mathrm{B}$ tied to a repressive protein, $\mathrm{I} \kappa \mathrm{B}$, which is decomposed by the action of certain cytokines, such as interleukin 1 (IL-1) and TNF- $\alpha$. Experiments have found that PPAR $\alpha$ agonists increase the intracellular concentration of $\mathrm{I} \kappa \mathrm{B}$ and thus repress the action of NFאB (17). Moreover, they are capable of regulating the expression of AP-1, which is also involved in the synthesis of proteins and inflammatory metabolites. It is believed that through this mechanism fibrate treatment is capable of diminishing the acute phase proteins, fibrinogens, and reactive-C protein in patients with heart disease. Other proteins, associated with the development of atherosclerotic plaque, also had decreased plasmatic concentrations when treated with PPAR $\alpha$ activators, such as IL-1, $-2,-6,-8$ and -12 (18). PPAR $\gamma$ is also associated with inflammation moderation, but this occurs through the suppression of STAT, AP-1 and NFKB by a trans-repression mechanism that is, as yet, not well explained.

During atherosclerotic plaque formation, endothelial cells and monocytes/macrophages exhibit pro-coagulating activity by tissular factor (TF) expression on the surface. PPAR $\alpha$ agonists reduce TF expression and the matrix metalloprotease in monocytes and macrophages, which potentially modifies the stability and thrombogenicity of the atherosclerotic lesion (19).

Other cells included in the pathogenesis of this illness are the T helper-1 linfocytes (LT-h1) which, when exposed to oxidized LDL, liberate certain proinflammatory cytokines, such as TNF- $\alpha$, INF- $\gamma$ and IL-2. These attract other cells to the vascular endothelium. PPAR $\alpha$ and PPAR $\gamma$ activators limit the expression of these cytokines, suggesting a modification of the inflammatory function of these transcription factors in the vascular wall (20).

Concerning the linfocyte $\mathrm{CD}^{+}$, several researchers have shown that the expression of proinflammatory cytokines is modified by PPAR $\gamma$ ligands. An example of this is the exposure of LT murine clones to glitazone and 15d-PGJ2, resulting in a reduction in IL-2 expression (21). However, the main factor related to LT-h1 expression is the inhibiting effect of the PPAR $\gamma$ agonists in dendritic cells where diminished IL-12 expression is found, an important factor that directs the response of these cells, especially during proliferation (20).

Fibrates are drugs that diminish blood levels of cholesterol and triglycerides, and permit an increase in HDL cholesterol. 
These metabolic effects are produced by the genetic activation of the genes controlled by this nuclear receptor, among which are apolipoprotein AI (ApoAI), apolipoprotein AII (ApoAII), lipoprotein lipase (LPL), adenosine triphosphate-binding cassette AI (ABCAI) and scavenger receptor class B type I (SRBI/CLAI). Another important effect of the PPAR $\alpha$ agonist is the reduction of the expression of the codifying gene for apolipoprotein CIII (ApoCIII), an LPL inhibitor (22-24).

The main use of fibrates in clinical practice is to reduce the level of triglycerides and to slightly raise the level of plasmatic HDL (25) by increasing LPL enzyme activity. This is done in two ways. First, LPL promoter gene transcription activity is increased, which has an element of response to PPAR (PPRE). Second, levels of ApoCIII, a natural inhibitor of LPL activity, are reduced (22). Another effect is the increase in size of the LDL macromolecules, which makes them less atherogenic than small particles (26). As previously mentioned, PPAR $\alpha$ activators increase the level of HDL, thus affecting cholesterol reverse transport from peripheral tissues to the liver, including the vascular endothelium. The mechanism includes an increase in the synthesis of ApoAI and ApoAII, the major protein components of HDL, in the liver. Moreover, PPAR $\alpha$ agonists induce the overexpression of ABCA1 transmembrane protein in macrophages, resulting in the efflux of cholesterol from these cells and initiating reverse cholesterol transport. This mechanism occurs indirectly through $\operatorname{LXR} \alpha$, which contains a PPRE region in its promoter (27).

Other drugs associated with the cholesterol reverse transport process are statins, which are also used in hypolipemiant therapy. Their action is to diminish the synthesis of cholesterol by the organism by inhibiting a key enzyme called hydroxymethylglutaryl CoA reductase (HMG CoA). This has also been observed, in clinical practices, to occasionally increase plasmatic levels of HDL. The way in which the statins behave is similar to that shown by the PPAR $\alpha$ agonists, increasing ABCA1 and ABCG1 expression (28), but this depends on the formation of geranyl-geranyl-PP RhoA based on or derived from mevalonate, a molecule which activates the PPAR $\alpha$ nuclear receptor by phosphorylation. This molecule remains inhibited by the intracellular reduction of mevalonate; in its inactive state it stimulates the presence of transcription factors, which go on to stimulate the appearance of PPAR $\gamma$, which, in turn, increases LXR $\alpha$ nuclear expression (29). In the same manner, PPAR $\alpha$ is activated, increasing the concentration of ApoAI (30).

The hypolipemiant properties of fibrates and the consequent reduction in cardiovascular disease are supported by many clinical studies including, among others, the Bezafibrate Coronary Atherosclerosis Intervention Trial (BECAIT), which researched the effect of bezafibrate and a controlled diet on atherosclerosis in patients, carriers of dyslipidemia (mainly hypertriglyceridemia), surviving severe myocardial infarction. Bezafibrate retarded the progression of focal atherosclerosis to a degree comparable with that reached by the statins, and reduced the incidence of coronary events in young postinfarction patients. As well, it brought about improvements in the lipid profile $(29,30)$.

The Bezafibrate Infarction Prevention (BIP) study investigated the effect of bezafibrate on patients with stable angina or prior infarction and high total cholesterol and evaluated the presence of fatal and non-fatal severe myocardial infarction. The probability of a coronary event was not significantly reduced, but a later analysis of a patient subgroup with high triglycerides revealed a significant reduction in the accumulated risk of coronary events $(29,32)$.

The Helsinki Heart Study (HHS) evaluated the reduction of the risk of coronary cardiopathy caused by high HDL cholesterol and the decrease in non-HDL cholesterol using gemfibrozil in asymptomatic middle-aged men with primary dyslipidemia. Marked improvement in the lipid profile in the group using gemfibrozil compared to the group using placebos was associated with a significantly reduced incidence of heart disease (29).

The Veterans Affairs High-Density Lipoprotein Cholesterol Intervention Trial (VA-HIT) examined the effect of gemfibrozil on non-fatal infarction and death caused by heart disease in men with coronary cardiopathy and low HDL. Gemfibrozil compared to placebos significantly reduced the risk of a coronary event. The risk of stroke was also significantly reduced. In spite of the fact that LDL levels in both groups were similar, the reduction in coronary events and death in the group treated with gemfibrozil was accompanied by an increase in HDL cholesterol and reduced total cholesterol and triglyceride levels. Even though an increase in HDL cholesterol is associated with reduced risk, this alone did not entirely explain the decrease, suggesting a pleiotropic effect for gemfibrozil $(29,33)$.

In spite of this evidence, cases have been reported in which the reduction of LDL levels was insufficient for the proposed objectives and, moreover, cases in which HDL cholesterol plasmatic concentration was also reduced. This information has led to the proposed hypothesis that there could be genetic variability in the information expressed by $\operatorname{PPAR} \alpha$, which would explain the different effects of these drugs. The most studied polymorphism of the PPAR $\alpha$ gene is that produced in codon 162, which leads to the substitution of a valine by a leucine $(\mathrm{L} 162 \mathrm{~V})$. There is recent research indicating that this polymorphism, among others, is capable of modifying nonclassic heart disease risk factors (34). Among individuals participating in the VA-HIT study, a relationship was demonstrated between the PPAR $\alpha$ polymorphism L162V and cardiovascular risk in patients with diabetes mellitus and insulin resistance (35). This same polymorphism affected ApoCIII and triglyceride concentration in patients participating in the Framingham Study (36), and was also associated with different components of metabolic syndrome with or without high-fat diets (37). The PPAR $\gamma$ polymorphism Pro 12A1a was associated with hydro-saline retention and edema found in some patients who were treated with agonists of this nuclear receptor (38). Similarly, there are many references regarding the topic of PPAR polymorphisms and their consequences.

\section{Fibrates and diabetes}

The Diabetes Atherosclerosis Intervention Study (DIAS) was designed to evaluate the effect of correcting lipoprotein abnormalities using fenofibrate on coronary atherosclerosis. Patients with good glycemic control, moderate lipoprotein alterations and at least one visible coronary lesion were randomly administered micronized fenofibrate or a placebo. 
The percentage of increase in the diameter of the stenosis and the decrease in minimal luminal diameter was significantly improved in the group treated with fenofibrate. The reduction in the angiographic progression of the coronary illness was related, at least in part, to significant changes in the levels of total cholesterol, HDL, LDL and triglycerides, even in subjects previously considered not to be in need of treatment (39-41).

The St. Mary's, Ealing, Northwick Park Diabetes Cardiovascular Disease Prevention (SENDCAP) study was set up to determine how intervention with fibrates (combined with conventional therapy) might improve cardiovascular response in patients with type 2 diabetes. Patients with no history of clinical cardiovascular disease were randomly administered either a placebo or bezafibrate. Those treated with bezafibrate had a significantly greater reduction in levels of triglycerides and, moreover, significantly greater increases in HDL cholesterol than in those given placebos. However, there was no significant difference in the advance of arterial disease as measured by ultrasound. The group receiving bezafibrate showed a significant reduction in the combined incidence of probable ischemic changes in the electrocardiogram at rest and in the incidence of infarction $(42,43)$.

The Fenofibrate Intervention and Event Lowering in Diabetes (FIELD) study was designed to evaluate the effect of fenofibrate on cardiovascular events in high-risk type 2 diabetes patients. The study was carried out on 9,795 patients with a 5-year (average) follow-up. The patients were randomly given a placebo or $200 \mathrm{mg}$ of fenofibrate and coronary events, including death from heart failure and non-fatal infarction, were evaluated. Fenofibrate did not reduce the incidence of fatal infarction, but there was a significant reduction (24\%) in non-fatal infarction and a non-significant reduction in total coronary events. There was no improvement in survival rate in the group treated with fenofibrate. The main finding of this study was the decrease in microvascular events. The relative risk of the necessity of revascularization was significantly reduced $(21 \%)$, the advance of albuminuric was slowed and the rate of diabetic retinopathy requiring laser treatment was reduced in comparison to the group receiving the placebo. It must be noted that an appreciable subgroup of the patients receiving placebos received treatment with statins (17 vs. $8 \%$ ) $(29,44,45)$.

Synthetic PPAR ligands are of particular interest in the treatment of type 2 diabetes since they reestablish insulin sensitivity. This property has been attributed to the direct effect of the PPAR agonists on the lipid metabolism in adipose tissue, and secondarily to their effect on glucose and the lipid metabolism in the liver and skeletal muscle. PPAR $\gamma$ agonists promote the differentiation of adipocytes and the capture and storing of free fatty acids in the subcutaneous adipose tissue rather than in the visceral tissue. This causes a reduction in free fatty acids and insulin resistance. Moreover, PPAR $\gamma$ activation increases the expression and transfer to the cell surface of the GLUT 1 and 4 glucose transporters, increasing muscular and hepatic glucose uptake and reducing plasmatic glucose levels. The PPAR $\gamma$ agonists reestablish insulin sensitivity, decreasing $\mathrm{TNF} \alpha$ expression and increasing adiponectin expression $(31,33,46-50)$. Furthermore, there are numerous studies demonstrating the direct effect of these drugs on pancreatic $\beta$ cells through a decrease in lipotoxicity over the pancreatic islets. How these function is not as yet clear, but the transcriptional repressor $\beta$-cell lymphoma-6 (BCL-6) plays a crucial role (51-55).

The effect of PPAR $\gamma$ is to stimulate the expression of the scavenger receptor (CD36), involved in the entrance of modified forms of cholesterol, among which are the 9 and 13-hydroxyoctadecadienoic (HODE) acids important in the pathogenesis of the atherosclerotic lesion (27). A hypothesis has been developed based on the fact that cholesterol exit from the macrophages is regulated by a transporter named ABCA 1 and in turn by PPAR $\gamma$. Thus, PPAR $\gamma$ regulates not only the entry but also the exit of cholesterol, from the macrophages to the liver, with HDL as the intermediary (27).

The therapeutic value of PPAR $\gamma$ agonists in the handling of insulin resistance has been demonstrated in a large number of studies with TZDs $(31,48,50,56)$.

Troglitazone was one of the first PPAR $\gamma$ agonists to be used, but was removed from the market due to serious hepatotoxic effects. In one study, troglitazone was administered in different doses to patients with type 2 diabetes. It was shown that levels of glycosylated hemoglobin $\left(\mathrm{HbA}_{\mathrm{IC}}\right)$, fasting glucose, plasmatic non-esterified fatty acids, triglycerides and fasting insulin were significantly lower in the group receiving treatment than in the control group. As well, the group receiving treatment showed an increase in insulin sensitivity, and HDL cholesterol was increased by the highest dose of troglitazone (29).

The Troglitazone in the Prevention of Diabetes (TRIPOD) study found that troglitazone improved insulin sensitivity in 2 out of 3 Hispanic women with prior pregnancy- related diabetes (59). Moreover, it protected against the development of type 2 diabetes through an action associated with the preservation of the functioning of pancreatic $\beta$ cells.

Later, studies on pioglitazone and rosiglitazone found no hepatotoxicity, confirming that this was not a class effect (57-59); however, similar metabolic effects were found. In a study using pioglitazone and a placebo control, $\mathrm{HbA}_{\mathrm{IC}}$, fasting glycemia, fasting insulin, $\mathrm{C}$ peptide and triglycerides were reduced while HDL cholesterol was increased. c-LDL and total cholesterol were not affected (60).

A recent study by the GLAI group investigated the effect of glitazones on lipid profiles. They determined that pioglitazone was associated with significant improvements in triglyceride, HDL and non-HDL cholesterol levels, and the size of LDL particles compared to rosiglitazone (50).

The PIOSTAT study demonstrated that pioglitazone and simvastatine have a very strong anti-inflammatory effect, as well as improving the homeostasis model assessment (HOMA) in patients with cardiovascular disease and high levels of ultra-sensitive C-reactive protein (CRP) (61).

The Pioglitazone Prevention of Type 2 Diabetes (PIPOD) study, an extension of the TRIPOD study, is currently investigating, by means of a 4-year follow-up, whether or not pioglitazone treatment offers protection against diabetes with normal or low glucose tolerance $(50,56)$.

As is the case with pioglitazone, the effect of rosiglitazone on the advance of diabetes is being investigated in various studies. The Diabetes Reduction Approaches with Ramipril and Rosiglitazone Medications (DREAM) study evaluated whether rosiglitazone alone or in combination with ramipril 
Table I. Known effects of PPAR agonists in the handling of insulin resistance.

Metabolic effects

Improvement and maintenance of glycemic control

Improvement in the metabolism of fatty acids

Improvement in lipid profile (increase of HDL with variable effect

on triglycerides)

Prevention of the progression of diabetes in high risk individuals
Stabilization of the function secretor of $\beta$ cells

Decrease in levels of inflammatory cytokines and protrombotic markers

Improvement in vascular physiology

Additional effects

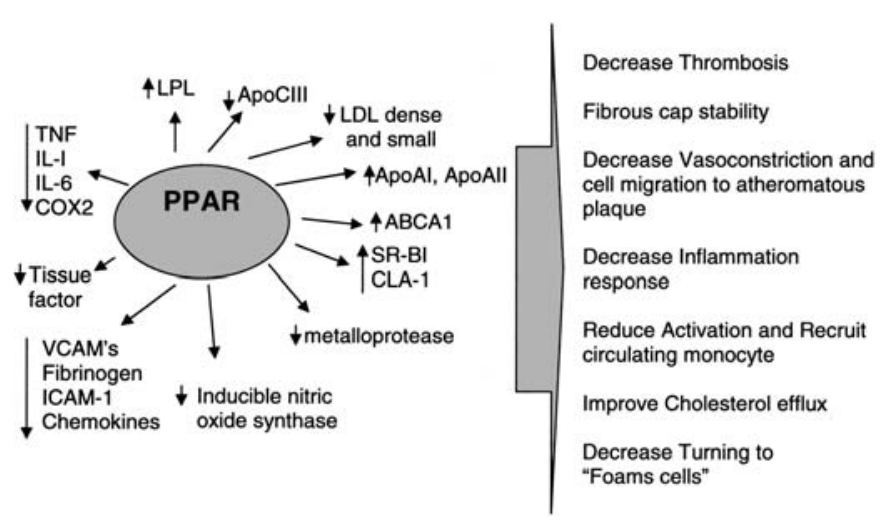

Figure 1. Pleiotropic effects of agonists of PPAR. LPL, lipoprotein lipase; IL-1, interleukin 1; IL-6, interleukin 6; COX-2, cyclooxygenase 2; ApoCIII, apoprotein CIII; ApoAI, apoprotein AI; ApoAII, apoprotein AII; SRB1/CLA-1, scavenger receptor class B type 1; ABCA1, cholesterol transport from family ABCA; ICAM-1, intercellular adhesion molecule-1; VCAM, vascular adhesion molecule-1.

is capable of slowing or preventing the advance of diabetes in subjects with glucose intolerance. The results were recently published and showed that, in spite of there being no significant differences between ramipril and the placebo for the advance of type 2 diabetes, rosiglitazone did achieve a reduction of over $50 \%$ in the progression from pre-diabetes to diabetes. Thus, this drug achieved the maximum return to normoglycemia in patients with glucose intolerance (62). Another study currently being carried out is the Canadian Normoglycemia Outcomes Evaluation (CANOE) study, which is evaluating whether treatment with rosiglitazone plus metformin can prevent the development of type 2 diabetes and reduce cardiovascular risk in patients with glucose intolerance (29).

\section{Beyond glycemic control}

In diabetics, the PPAR agonists have demonstrated many other beneficial effects apart from those having to do with insulin resistance and glucide metabolism.

Over $70 \%$ of diabetics have some degree of dyslipidemia, usually characterized by elevated levels of triglycerides and low HDL. The United Kingdom Prospective Diabetes Study (UKPDS) demonstrated that lipid abnormalities are an even greater risk factor for coronary cardiopathy than $\mathrm{HbA}_{\mathrm{IC}}$ (63), a highly significant fact given that nearly $80 \%$ of type 2 diabetics die from cardiovascular complications. The ability of the PPAR $\alpha$ agonists, like fibrates, to improve the lipid profile is well established. As well, there is evidence that PPAR $\gamma$ has a positive impact on diabetic dyslipidemia $(29,31,46)$.

Type 2 diabetes is also characterized by elevated levels of inflammatory markers such as CRP, which is intimately tied to the risk of cardiovascular disease (64).

Taking advantage of their beneficial effect on glycemia, the lipid profile and their anti-inflammatory effects, a new group of PPAR $\alpha / \gamma$ dual agonists are being investigated. A few, such as ragaglitazar and MK-0767, were removed from the market because of their toxicity $(29,31,33,49)$.

Preliminary findings in an ongoing study with muraglitazar are discouraging. In this study, different doses of muraglitazar, pioglitazone or placebo are being compared in monotherapy or in combination with metformin or glyburide to evaluate their effect on death and major cardiovascular events in patients with type 2 diabetes. Compared with the placebo or pioglitazone, muraglitazar has been associated with an excessive incidence of death, major cardiovascular events and cardiac insufficiency (65).

On the other hand, a recently published study on muraglitazar and pioglitazone, either alone or in combination with metformin, showed a significant improvement in $\mathrm{HbA}_{\mathrm{IC}}$ and in lipid profiles (66).

Another ongoing study with tesaglitazar has shown encouraging preliminary results, decreasing fasting glycemia and triglycerides and increasing HDL with a low percentage of adverse effects (67).

\section{Potential applications of PPAR agonists}

Along with the positive effects of glitazones on glycemia and fibrates on dyslipidemia, the PPAR agonists are seen as being able to greatly influence many other components of metabolic syndromes, such as hypertension, inflammation, vascular dysfunction and vascular remodeling (Table I). The latter are also seen in atherosclerosis, where the effects of the PPAR pleiotropics have an added value in the treatment of cardiovascular complications $(30,32,47)$ (Fig. 1).

Studies directed toward determining whether glitazones have beneficial long-term effects at the cardiovascular level are currently underway.

The Prospective Pioglitazone Clinical Trial in Macrovascular Events (PROACTIVE) was directed toward proving whether treatment with pioglitazone reduces the incidence of macrovascular illness and consequent death in high risk type 2 diabetic patients. This recently published trial, involving 
5,238 patients in a 34.5-month (average) follow-up, demonstrated that treatment with pioglitazone reduced death from all causes, the incidence of non-fatal infarction and strokes in diabetics with a high risk of macrovascular events $(44,56)$.

As well, there are studies that aim to demonstrate the antiinflammatory properties of the PPAR agonists on endothelial function and vascular remodeling, as well as its other effects at the macrovascular level.

One study, with 357 type 2 diabetics tracked over 26 weeks, determined that the use of rosiglitazone significantly reduced the levels of CRP and also brought down the levels of metalloprotease, a plaque stability marker, in the extracellular matrix (65). In another study of non-diabetic patients with coronary cardiopathy, it was shown that rosiglitazone reduced not only inflammation markers but also the endothelial activation markers E-selectin, Von Willebrand factor, fibrinogen and CRP (66).

PPAR $\gamma$ is strongly associated with adipose differentiation, from undifferentiated fibroblast-type adipose to the appearance of a small, mature adipose cell. Knockout mice (-/-) for PPAR $\gamma$ die in utero due to problems with the placenta; heterozygotes $(-/+)$ show increased sensitivity to insulin and protection from obesity when they are exposed to high-fat diets. The ligands most used in these experiments were TZDs such as rosiglitazone and pioglitazone. Treatment with TZDs or glitazones produced hypoglycemiant effects and improved sensitivity to insulin. Other functions included the storing of lipids, not only in the liver but also in the adipose tissue, and the regulation of the expression of adipokines (67). In addition to these results, a protein called PPAR $\gamma$ coactivator $-1 \alpha$, (PGC-1 $\alpha$ ), related to the activation of this transcription factor, produced a powerful set of genetic responses to changes occurring in the cell environment once stimulated, such as increased uptake of glucose increasing transporter expression in different tissues and the control of hepatic gluconeogenesis (68).

Arterial hypertension is also affected by the PPAR $\gamma$ activators which, like PPAR $\alpha$, inhibit the expression of endothelin (ET)-1, a potent vasoconstrictor that reduces the production of free radicals under treatment with glitazone (70).

It has recently been discovered that PPAR $3 / \delta$ has a special relevance to the control of fatty acid catabolism and energy homeostasis, confirmed by the use of the synthetic agonist GW 501506. The studies point to PPAR isoforms as a key element in the use of fatty acid by cells and in the coordination of energy use (70).

A very recent study demonstrated that the effect of rosiglitazone on muscle sensitivity to insulin is produced by PPAR $\delta$ and the PGC- $1 \alpha$ coactivator, and is dependent on the improved oxidative capacity of the muscle (71).

The benefits of the PPAR agonists are many. Nevertheless, as with all medication, TZDs and fibrates carry undesirable effects, such as hydrosaline retention, that may lead to edema (72) and lack compensation in congestive illnesses (73). It is worth pointing out that this effect is not observed with statins (72). Another undesirable effect is weight gain in patients receiving TZD and fibrates (74-76), similar to observations in the majority of glycemia-reducing therapies.

Finally of note is the recent discovery that telmisartan, a drug that blocks the angiotensin receptor (77) and has been used in the treatment of hypertension, depends on PPAR $\gamma$ for activation. Telmisartan improves insulin sensitivity in nondiabetic patients with hypertension $(78,79)$ and, moreover, induces adipogenesis via PPAR $\gamma(80)$. It also inhibits the production of CRP in hepatocytes (81) and improves metabolic parameters, such as $\mathrm{HbA}_{\mathrm{IC}}$ and blood pressure, in diabetic and hypertensive patients (82-85).

\section{Conclusions}

In light of the previous information, it is evident that PPARs, with their isoforms, polymorphisms and ligands both natural and synthetic, constitute a tremendously important group in terms of their potential role as targets for drug treatment. This role takes on even greater relevance in modern societies, where there is a growing incidence of illnesses such as obesity, diabetes and cardiovascular disease, for the most part related to the excessive accumulation of fats. This is due in large part to unhealthy, sedentary lifestyles with high calorie intake, as well as to genetic alterations in energy metabolism.

Undoubtedly, a change of lifestyle is the basis of any treatment of these pathologies, but the lack of adherence to this measure constitutes a major challenge to the drug industry. Beyond the discussion of cost versus the benefit of using rosiglitazone in the prevention of diabetes, and the lack of class evidence which backs up this use and/or its use in cardiovascular disease prevention, this family of nuclear receptors constitutes a new superfamily that, given its potential efficacy, should be considered when studying and treating diseases associated with glucose metabolism, lipids, and atherosclerosis. Recent studies have shown that roziglitazone is also likely to have an effect on cardiovascular disease (86).

A final point regarding PPAR agonists is their pleiotropic effect, by which they are capable of combating illnesses developed due to the disruption produced by hypercholesterolism. They do so not only by decreasing levels of plasmatic cholesterol, influencing the pathological profile of LDL by reducing its size, but also by also inhibiting the inflammatory process which takes place in the vascular epithelium during the development of atherosclerotic lesions.

\section{References}

1. Lalwani ND, Reddy MK, Qureshi SA, et al: Evaluation of selected hypolipidemic agents for the induction of peroxisomal enzymes and peroxisome proliferation in the rat liver. Hum Toxicol 2: $27-48,1983$

2. Desvergne B and Wahli W: Peroxisome proliferator-activated recptor: Nuclear control of metabolism. Endocr Rev 20: 649-688, 1999.

3. Juge-Aubry CE, Hammar E, Siegrist-Kaiser C, et al: Regulation of the transcriptional activity of the peroxisome proliferator-activated receptor alpha by phosphorylation of a ligand-independent transactivating domain. J Biol Chem 274: 10505-10510, 1999.

4. Wahli W, Braissant O and Desvergne B: Peroxisome proliferator activated receptors: transcriptional regulators of adipogenesis, lipid metabolism and more. Chem Biol 2: 261-266, 1995.

5. Braissant O, Foufelle F, Scotto C, Dauca M and Wahli W: Differential expression of peroxisome proliferator-activated receptors (PPARs): tissue distribution of PPAR $\alpha, \beta$ and $\gamma$ in the adult rat. Endocrinology 137: 354-366, 1996.

6. Schoonjans K, Staels B and Auwerx J: Role of the peroxisome proliferator-activated receptor (PPAR) in mediating the effects of fibrates and fatty acids on gene expression. J Lipid Res 37: 907-925, 1996. 
7. Lee S, Pineau T, Drago J, Lee E, Owens J, Kroetz D, FernandezSalguero P-M, Westphal H and Gonzalez F: Targeted disruption of the $\alpha$ isoform of the peroxisome proliferator-activated receptor gene in mice results in abolishment of the pleiotropic effects of peroxisome proliferators. Mol Cell Biol 15: 3012-3022, 1995.

8. Knauf C, Rieusset J, Foretz M, et al: Peroxisome proliferatoractivated receptor-alpha-null mice have increased white adipose tissue glucose utilization, GLUT4, and fat mass: Role in liver and brain. Endocrinology 147: 4067-4078, 2006.

9. Cuzzocrea S, Mazzon E, Di Paola R, et al: The role of the peroxisome proliferator-activated receptor-alpha (PPAR-alpha) in the regulation of acute inflammation. J Leukoc Biol 79: 999-1010, 2006.

10. Delayre-Orthez C, Becker J, Guenon I, et al: PPAR alpha downregulates airway inflammation induced by lipopolysaccharide in the mouse. Respir Res 6: 91-101, 2005.

11. Li S, Gokden N, Okusa MD, et al: Anti-inflammatory effect of fibrate protects from cisplatin-induced ARF. Am J Physiol Renal Physiol 289: 469-480, 2005.

12. Loichot C, Jesel L, Tesse A, et al: Deletion of peroxisome proliferator-activated receptor-alpha induces an alteration of cardiac functions. Am J Physiol Heart Circ Physiol 291: 161-166, 2006.

13. Guerre-Millo M, Rouault C, Poulain P, et al: PPAR-alpha-null mice are protected from high-fat diet-induced insulin resistance. Diabetes 50: 2809-2814, 2001.

14. Marx N, Kehrle B, Kohlhammer K, et al: PPAR activators as antiinflammatory mediators in human T lymphocytes: implications for atherosclerosis and transplantation-associated arteriosclerosis. Circ Res 90: 703-710, 2002

15. Devchand PR, Keller H, Peters JM, Vazquez M, Gonzalez FJ and Wahli W: The PPAR $\alpha$ leukotriene B4 pathway to inflammatory control. Nature 384: 39-43, 1996

16. Marx N, Sukhova GK, Collins T, Libby P and Plutzky J: PPAR $\alpha$ activators inhibit cytokine-induced vascular cell adhesion molecule-1 expression in human endothelial cells. Circulation 99: 3125-3131, 1999.

17. Kleeman R, Gervois PP, Verschuren L, et al: Fibrates downregulate IL-1-stimulated $\mathrm{C}$-reactive protein gene expression in hepatocytes by reducing nuclear p50-NFкB-C/EBP-ß complex formation. Blood 101: 545-551, 2003.

18. Sun $\mathrm{Z}$ and Andersson R: NFkB activation and inhibition: a review. Shock 18: 99-106, 2002.

19. Neve BP, Corseaux D, Chinetti G, et al: PPAR $\alpha$ agonists inhibit tissue factor expression in human THP-1 cells, monocytes and macrophages. Circulation 103: 207-212, 2001.

20. Faveeuw C, Fougeray S, Angeli V, et al: Peroxisome proliferatoractivated receptor $\gamma$ activators inhibit interleukin-12 production in murine dendritic cells. FEBS Lett 486: 261-266, 2000.

21. Clark RB, Bishop Bailey D, Estrada Hernandez T, Hla T, Puddington L and Padula SJ: The nuclear receptor PPAR $\gamma$ and immunoregulation: PPAR $\gamma$ mediates inhibition of helper $\mathrm{T}$ cell responses. J Immunol 164: 1364-1371, 2000.

22. Staels B, Vu-Dac N, Kosykh VA, et al: Fibrates downregulate apolipoprotein C-III expression independent of induction of peroxisomal acyl coenzyme A oxidase. A potential mechanism for the hypolipidemic action of fibrates. J Clin Invest 95: 705-712, 1995 .

23. Torra IP, Chinetti G, Duval C, Fruchart JC and Staels B: Peroxisome proliferator-activated receptors: from transcriptional control to clinical practice. Curr Opin Lipidol 12: 245-254, 2001.

24. De Silva HV, Lauer SJ, Wang J, et al: Overexpression of human apolipoprotein C-III in transgenic mice results in an accumulation of apolipoprotein B48 remnants that is corrected by excess apolipoprotein E. J Biol Chem 269: 2324-2335, 1994.

25. Chapman MJ: Fibrates in 2003: therapeutic action in atherogenic dyslipidemia and future perspectives. Atherosclerosis 171: $1-13,2003$.

26. Yoshida H, Ishikawa T, Ayaori M, et al: Beneficial effect of gemfibrozil on the chemical composition and oxidative susceptibility of low density lipoprotein: a randomized, doubleblind, placebo-controlled study. Atherosclerosis 139: 179-187, 1998 .

27. Chinetti G, Lestavel S, Bocher V, et al: PPAR- $\alpha$ and PPAR- $\delta$ activators induce cholesterol removal from human macrophage foam cells through stimulation of the ABCA1 pathway. Nat Med 7: 53-58, 2001

28. Argmann C, Edwards J, Sawyez C, et al: Regulation of macrophage cholesterol efflux through hydroxymethylglutarylCoA reductase inhibition. J Biol Chem 280: 22212-22221, 2005.
29. Staels B and Fruchart JC: Therapeutic roles of peroxisome proliferator-activated receptor agonists. Diabetes 54: 2460-2470, 2005.

30. Martin G, Duez H, Blanquart C, et al: Statin-induced inhibition of the Rho-signaling pathway activates PPARa and induces HDL apoA-I. J Clin Invest 107: 1423-1432, 2001.

31. Tenenbaum A, Fisman E and Motro M: Metabolic syndrome and type 2 diabetes mellitus: focus on peroxisome proliferator activated receptors. Cardiovasc Diabetol 2: 4-11, 2003.

32. The BIP Study Group: Secondary prevention by raising HDL cholesterol and reducing triglycerides in patients with coronary artery disease The Bezafibrate Infarction Prevention (BIP) Study. Circulation 102: 21-27, 2000

33. Willa AH and Bruemmer D: Peroxisome proliferator-activated receptor: Implications for cardiovascular disease. Hipertensión 43: 297-305, 2004

34. Manresa JM, Zamora A, Tomas M, et al: Relationship of classical and non-classical risk factors with genetic variants relevant to coronary heart disease. Eur J Cardiovasc Prev Rehabil 13 738-744, 2006.

35. Tai ES, Collins D, Robins SJ, et al: The L162V polymorphism at the peroxisome proliferator activated receptor alpha locus modulates the risk of cardio-vascular events associated with insulin resistance and diabetes mellitus: the Veterans Affairs HDL Intervention Trial (VA-HIT). Atherosclerosis 187: 153-160, 2006.

36. Tai ES, Corella D, Demissie S, et al: Framingham Heart Study. Polyunsaturated fatty acids interact with the PPARA-L162V polymorphism to affect plasma triglyceride and apolipoprotein C-III concentrations in the Framingham Heart Study. J Nutr 135: 397-403, 2005.

37. Robitaille J, Brouillette C, Houde A, et al: Association between the PPARalpha-L162V polymorphism and components of the metabolic syndrome. J Hum Genet 49: 482-489, 2004.

38. Hansen L, Ekstrom CT, Tabanera Y, et al: The Pro12Ala variant of the PPARG gene is a risk factor for peroxisome proliferatoractivated receptor-gamma/alpha agonist-induced edema in type 2 diabetic patients. J Clin Endocrinol Metab 91: 3446-3450, 2006.

39. Ansquer JC, Foucher C, Rattier S, Taskinen MR and Steiner G: DAIS Investigators. Fenofibrate reduces progression to microalbuminuria over 3 years in a placebo-controlled study in type 2 diabetes: results from The Diabetes Atherosclerosis Intervention Study (DAIS). Am J Kidney Dis 45: 485-493, 2005.

40. Vakkilainen J, Steiner G, Ansquer JC, et al: DAIS group. Relationships between low-density lipoprotein particle size, plasma lipoproteins, and progression of coronary artery disease: the Diabetes Atherosclerosis Intervention Study (DAIS). Circulation 107: 1733-1737, 2003.

41. McLaughlin PR and Gladstone P: Diabetes Atherosclerosis Intervention Study (DAIS): quantitative coronary angiographic analysis of coronary artery atherosclerosis. Cathet Cardiovasc Diagn 44: 249-256, 1998

42. Elkeles RS, Diamond JR, Poulter C, et al: Cardiovascular outcomes in type 2 diabetes. A double-blind placebo-controlled study of bezafibrate: The St. Mary's, Ealing, Northwick Park Diabetes Cardiovascular Disease Prevention (SENDCAP) Study. Diabetes Care 21: 641-648, 1998.

43. Elkeles RS, Diamond JR, El-Bahghouti N, et al: Relative fasting hypoinsulinaemia and ultrasonically measured early arterial disease in type 2 diabetes. The SENDCAP Study Group, St. Mary's, Ealing, Northwick Park Diabetes Cardiovascular Disease Prevention Study. Diabet Med 13: 247-253, 1996.

44. Robinson JG: Update on PPAR agonists: The clinical significance of FIELD and PROACTIVE. Curr Atheroscler Rep 9: 64-71, 2007.

45. The FIELD study investigators. Effects of long-term fenofibrate therapy on cardiovascular events in 9795 people with type 2 diabetes mellitus (The FIELD Study): randomised controlled trial. Lancet 366: 1849-1861, 2005.

46. Barbier O, Pineda Torra I, Duguay Y, et al: Pleiotropic actions of peroxisome proliferator-activated receptors in lipid metabolism and atherosclerosis. Arterioscler Thromb Vasc Biol 22: 717-726, 2002.

47. Yki-Järvinen H: Thiazolidinediones. N Engl J Med 351: 1106-1118, 2004

48. Inzucchi S: Oral antihyperglycemic therapy for type 2 diabetes. JAMA 287: 360-372, 2002.

49. Vamecq $J$ and Latruffe N: Medical significance of peroxisome proliferator-activated receptors. Lancet 354: 141-148, 1999. 
50. Goldberg R, Kendall D, Deeg M, et al: A comparison of lipid and glycemic effect of pioglitazone and rosiglitazone in patients with type 2 diabetes mellitus and dyslipidemia. Diabetes Care 28: 1547-1554, 2005.

51. Kharroubi I, Lee CH, Hekerman P, et al: BCL-6: a possible missing link for anti-inflammatory PPAR-delta signalling in pancreatic beta cells. Diabetologia 49: 2350-2358, 2006.

52. Lalloyer F, Vandewalle B, Percevault F, et al: Peroxisome proliferator-activated receptor alpha improves pancreatic adaptation to insulin resistance in obese mice and reduces lipotoxicity in human islets. Diabetes 55: 1605-1613, 2006.

53. Kawai T, Hirose H, Seto Y, et al: Troglitazone ameliorates lipotoxicity in the beta cell line INS-1 expressing PPAR gamma. Diabetes Res Clin Pract 56: 83-92, 2002.

54. Harrity T, Farrelly D, Tieman A, et al: Muraglitazar, a novel dual (alpha/gamma) peroxisome proliferator-activated receptor activator, improves diabetes and other metabolic abnormalities and preserves beta-cell function in $\mathrm{db} / \mathrm{db}$ mice. Diabetes 55: 240-248, 2006.

55. Welters HJ, McBain SC, Tadayyon M, Scarpello JH, Smith SA and Morgan NG: Expression and functional activity of PPARgamma in pancreatic beta cells. Br J Pharmacol 142: 1162-1170, 2004.

56. Dormandy J, Charbonnel B, Eckland DJ, et al on behalf of the PROactive investigators: Secondary prevention of macrovascular events in patients with type 2 diabetes in the PROactive Study (Prospective Pioglitazone Clinical Trial in Macrovascular Events): a Randomised Controlled Trial. Lancet 366: 1279-1289, 2005.

57. Snitker S, Watanabe RM, Ani I, et al: Troglitazone in Prevention of Diabetes (TRIPOD) Study. Changes in insulin sensitivity in response to troglitazone do not differ between subjects with and without the common, functional Pro12Ala peroxisome proliferatoractivated receptor-gamma2 gene variant: results from the Troglitazone in Prevention of Diabetes (TRIPOD) Study. Diabetes Care 27: 1365-1368, 2004

58. Ratner RE: The Diabetes Prevention Program Research. An update on the Diabetes Prevention Program. Endocr Pract 12: 20-24, 2006.

59. Knowler WC, Hamman RF, Edelstein SL, et al: Diabetes Prevention Program Research Group. Prevention of type 2 diabetes with troglitazone in the Diabetes Prevention Program. Diabetes 54: 1150-1156, 2005.

60. Pfutzner A, Schneider CA and Forst T: Pioglitazone: an antidiabetic drug with cardiovascular therapeutic effects. Expert Rev Cardiovasc Ther 4: 445-459, 2006.

61. Hanefeld M, Marx N, Pfutzner A, et al: Anti-inflammatory effects of pioglitazone and/or simvastatin in high cardiovascular risk patients with elevated high sensitivity C-reactive protein: The PIOSTAT Study. J Am Coll Cardiol 49: 290-297, 2007.

62. DREAM Trial Investigators, Bosch J, Yusuf S, Gerstein HC, Pogue J, Sheridan P, Dagenais G, Diaz R, Avezum A, Lanas F, Probstfield J, Fodor G and Holman RR: Effect of ramipril on the incidence of diabetes. N Engl J Med 355: 1551-1562, 2006.

63. Stevens RJ, Coleman RL, Adler AI, Stratton IM, Matthews DR and Holman RR: Risk factors for myocardial infarction case fatality and stroke case fatality in type 2 diabetes: UKPDS 66. Diabetes Care 27: 201-217, 2004.

64. Pfutzner A and Forst T: High-sensitivity C-reactive protein as cardiovascular risk marker in patients with diabetes mellitus. Diabetes Technol Ther 8: 28-36, 2006.

65. Nissen S, Wolsky K and Topol E: Effect of muraglitazar on death and major adverse cardiovascular events in patients with type 2 diabetes mellitus. JAMA 294: 2581-2586, 2005.

66. Kendall DM, Rubin CJ, Mohideen P, et al: Improvement of glycemic control, triglycerides, and HDL cholesterol levels with muraglitazar, a dual (alpha/gamma) peroxisome proliferatoractivated receptor activator, in patients with type 2 diabetes inadequately controlled with metformin monotherapy: a doubleblind, randomized, pioglitazone-comparative study. Diabetes Care 29: 1016-1023, 2006

67. Goldstein BJ, Rosenstock J, Anzalone D, Tou C and Ohman KP: Effect of tesaglitazar, a dual PPARalpha/gamma agonist, on glucose and lipid abnormalities in patients with type 2 diabetes: a 12-week dose-ranging trial. Curr Med Res Opin 22: 2575-2590, 2006.
68. Marx N, Froehlich J, Siam L, et al: Anti-diabetic PPAR gammaactivator rosiglitazone reduces MMP-9 serum levels in type 2 diabetic patients with coronary artery disease. Arterioscler Thromb Vasc Biol 23: 283-288, 2003.

69. Sidhu JS, Cowan D and Kaski JC: The effects of rosiglitazone, a peroxisome proliferator-activated receptor-gamma agonist, on markers of endothelial cell activation, C-reactive protein, and fibrinogen levels in non-diabetic coronary artery disease patients. J Am Coll Cardiol 42: 1757-1763, 2003

70. Evans R, Grant B and Wang XY: PPARs and the complex jouney to obesity. Nat Med 10: 1-7, 2004.

71. Yoon JC, Puigserver P, Chen G, et al: Control of hepatic gluconeogenesis through the transcriptional coactivator PGC-1. Nature 413: 131-138, 2001.

72. Iglarz M, Touyz RM, Amiri F, Lavoie MF, Diep QN and Schiffrin EL: Effect of peroxisome proliferator-activated receptor- $\alpha$ and $-\gamma$ activators on vascular remodeling in endothelindependent hypertension. Arterioscler Thromb Vasc Biol 23: $45-51,2003$

73. Wang YX, Lee CH, Tiep S, Yu RT, Ham J, Kang H and Evans RM: Peroxisome proliferators-activated receptor $\delta$ activates fat metabolism to prevent obesity. Cell 113: 159-170, 2003.

74. Mensink M, Hesselink MK, Russell AP, Schaart G, Sels JP and Schrauwen P: Improved skeletal muscle oxidative enzyme activity and restoration of PGC-1 alpha and PPARbeta/delta gene expression upon rosiglitazone treatment in obese patients with type 2 diabetes mellitus. Int J Obes 31: 1302-1310, 2007.

75. Shiroshita-Takeshita A, Brundel BJ, Burstein B, et al: Effects of simvastatin on the development of the atrial fibrillation substrate in dogs with congestive heart failure. Cardiovasc Res 74: 75-84, 2007.

76. Srivastava PM, Calafiore P, MacIsaac RJ, Hare DL, Jerums G and Burrell LM: Thiazolidinediones and congestive heart failure exacerbation or new onset of left ventricular dysfunction? Diabet Med 21: 945-950, 2004

77. Nichols GA and Gomez-Caminero A: Weight changes following the initiation of new anti-hyperglycaemic therapies. Diabetes Obes Metab 9: 96-102, 2007.

78. Chiang CK, Ho TI, Peng YS, et al: Rosiglitazone in diabetes control in hemodialysis patients with and without viral hepatitis infection: effectiveness and side effects. Diabetes Care 30: 3-7, 2007.

79. Fonseca V: Effect of thiazolidinediones on body weight in patients with diabetes mellitus. Am J Med 115: 42S-48S, 2003.

80. Schupp M, Clemenz M, Gineste R, et al: Molecular characterization of new selective peroxisome proliferator-activated receptor gamma modulators with angiotensin receptor blocking activity. Diabetes 54: 3442-3452, 2005.

81. Benndorf RA, Rudolph T, Appel D, et al: Telmisartan improves insulin sensitivity in nondiabetic patients with essential hypertension. Metabolism 55: 1159-1164, 2006.

82. Erbe DV, Gartrell K, Zhang YL, et al: Molecular activation of PPARgamma by angiotensin II type 1-receptor antagonists. Vascul Pharmacol 45: 154-162, 2006.

83. Janke J, Schupp M, Engeli S, et al: Angiotensin type 1 receptor antagonists induce human in-vitro adipogenesis through peroxisome proliferator-activated receptor-gamma activation. J Hypertens 24: 1809-1816, 2006.

84. Yoshida T, Yamagishi S, Nakamura K, et al: Telmisartan inhibits AGE-induced C-reactive protein production through downregulation of the receptor for AGE via peroxisome proliferator-activated receptor-gamma activation. Diabetologia 49: 3094-3099, 2006

85. Negro R and Hassan H: The effects of telmisartan and amlodipine on metabolic parameters and blood pressure in type 2 diabetic, hypertensive patients. J Renin Angiotensin Aldosterone Syst 7: 243-246, 2006.

86. Nissen SE and Wolski K: Effect of rosiglitazone on the risk of myocardial infarction and death from cardiovascular causes. $\mathrm{N}$ Engl J Med 356: 2457-2471, 2007. 